\title{
Apport de la physico-chimie à l'étude du retrait-gonflement d'une argile plastique compactée
}

\section{H. SOULI,}

J.-M. FLEUREAU

Laboratoire MSSMat,

CNRS UMR8579

et Ecole centrale Paris

Grande Voie des Vignes 92295 Châtenay-Malabry cedex

Hanene.Soulioecp.fi

Jean-Marie.Fleureau@ecp. $f^{*}$

\section{R. SOEMITRO}

Jurusan Teknix Sipil, Institut Tekrologi Sepuluh Nopember Kampus ITS-Sukolilo

Surabaya 60111 (Indonésie) soernitro@sby.dnet.net.id
L'article présente le comportement d'une argile très plastique $\left(w_{\mathrm{L}}=170 \%, \mathrm{I}_{\mathrm{P}}=110 \%\right)$, compactée à l'optimum Proctor normal, sur les chemins de drainage et d'humidification. La pression de gonflement a été mesurée par deux méthodes, avec des valeurs de 540 et $880 \mathrm{kPa}$. Les essais de drainage-humidification effectués sur des échantillons normalement consolidés, surconsolidés et compactés mettent en évidence l'effet de l'état initial. Sur le chemin de drainage, l'échantillon compacté présente un comportement surconsolidé tandis que, sur le chemin d'htimidification, son gonflement est moins important que celui des matériaux normalement consolidés ou surconsolidés séchés. Pour comprendre ces différences, des mesures physico-chimiques ont été effectuées afin d'étucier le rôle des différentes catégories de pores ainsi que l'évolution de l'organisation et de la texture du matériau lors du gonflement. La porosimetrie au mercure et l"analyse thermique mettent en évidence le ròle prépondérant des pores interagrégats dans le processus de gonflement alors que la diffraction des rayons $X$ indique que le nombre de couches d'eau adsorbées change relativement peu. La comparaison des formes des réflexions (001) permet en outre d'avoir une idée qualitative sur l'orientation des particules. Ces differentes analyses constituent un apport tres important pour expliquer les observations macroscopiques.

Mots-çés : retrait, gonflement, pression de gonflement, compactage Proctor, texture, parosité, physico-chimie.

\section{Contribution of physicochemistry to the study of the swelling-shrinkage behaviour of a compacted plastic clay}

The paper presents a study of the hydromechanical behaviour of a very plastic $\left(w_{1}=170 \%, I_{p}=110 \%\right.$ ) clay sample, compacted to the Proctor optimin, on drying-wetting paths. The swe'ling pressure value derived from two methods varies from $540 \mathrm{kPa}$ to $890 \mathrm{kPa}$. Drying and wetting tests carried out on normally consolidated, overconsolidated and compacted clay samples highlight the influence of the intial state. On drying path, the compacted sample exhibits arn overconsolidated behaviour whereas, on wetting path, its sweiling is smaller than that of normally consolidated and overconsolidated dried specimens. To understand these differences, physicochemical tests were carried out to study the contribution of the different classes of pores to the swelling process, as well as the changes in the organization and fabric of the sample. Mercury intrusion porosimetry and thermal analysis highight the predominant part of the interaggregate pores in the swelling process. X-ray diffraction shows that the number of layers of adsorbed water does not change very much during wetting. Fusthermare, the comparison of the shapes of the (001) reflections gives a qualitetive idea about particle orientation. These different analyses bring out an important contribution to explain the macroscopic observations. 


\section{Introduction}

Les argiles suscitent beaucoup d'intérêt dans différentes applications. Leurs diverses propriétés, notamment leur capacité de gonflement et de rétention, font d'elles des matériaux intéressants dans de nombreux domaines: fluides de forage pour le génie civil et le génie pétrolier, additifs poum les industries pharmaceutique et cosmétique, barrières étanches pour lè stockage des déchets, etc. En revanche, ces mêmes caractéristiques font que les argiles et les sols argileux sont redoutés en tant que matériaux de fondation des bấtinents ou matériaux de remblais. En par'iculier, la construction des ouvages sur des sols argileux nécessite de caractériser le potentiel de retrait-gonflement de ces sols. Ainsi, un sol argileux très plastique humidifié sous de faibles contraintes pourra gonfler fortement et son séchage se traduira par une importante diminution de volume. Plusieurs études se sont intéressées aux facteurs influençant les changements volumiques des argiles: Chen (1988) relie le gonflement des argiles à leur limite de liquidité, Komine et Ogata (1994) et Suratman (1995) montrent que le taux de gonflement varie linéairement en fonction de la densité sèche initiale tandis que la pression de gonflement décrit une loi exponentielle en fonction de la densité sèche initiale. Hachichi et Fleureau (1999) trouvent que le gonflement augmente quand la teneur en eau initiale du sol diminue, mais seulement lorsque celle-ci est supérieure à la limite de retrait. Is montrent en outre que la pression de gonflement suit une loi lineaire en fonction de la teneur en eau. Plusieurs études réalisées dans le domaine des sols non saturés (Biarez et al, 1993, 1994 ; Fleureau et al., 1995; Modaressi et al., 1996) ont permis de développer des modèles globaux de comportement basés sur le concept de contraintes effectives. Dans une autre approche, Fleureau et al. (1993) ont étudié les courbes de drainage-humidification de sols de différentes minéralogies pris dans divers états de consolidation en essayant de relier certaines caractéristiques des sols comme la limite de retrait ou la pression de désaturation à la himite de liquidité. Les auteurs ont aussi montré que les courbes de drainage étaient sensibles à l'état initial du sol et au mode de préparation des échantillons.

L'objectif de l'article est de présenter différents aspects du retrait-gonflement d'une argile très plastique compactée à loptimum Proctor nomal (OPN), aux échelles macroscopique et microscopique. Dans la premièrc partie, la pression de gonflement de l'argile est mesurée par les méthodes d'humidification sous différentes contraintes et de gonflement libre-rechargement, sont étudiées les variations de volume et de saturation de l'échantillon lors d"un cycle de drainagehumidification. Cette première partie met en évidence une différence de comportement entre le sol compacté et les sols normalement consolidés et surconsolidés, pris comme références, sur le chemin d'humidification. Pour expliquer ce résultat, différentes techniques physico-chimiques ont été mises en neuvre dans la seconde partie de l'étude, en vue d'analyser les phénomènes à une échelle plus petite. Ces techniques, appliçuées à l'élude du gonllement de l'échantillon d'argile compacté à l'OPN, mettent en évidence les transformations subies par le matériau du point de vue de la porosité et de la texture. Ainsi, la diffraction des rayons X donne accès aux distances interfoliaires et à l'eau adsorbête, tandis que la comparaison des formes des réflexions (001) permet d'avoir une idée qualitative sur l'orientation des particules et l'anisotropie du matériau. La porosimétrie au mercure et l'analyse themique permettent d'étudier la contribution des différentes classes de macropores au processus de gonflement sous l'effet du réarrangement des particules.

\section{2}

\section{Méthodes expérimentales}

La mesure des limites d'Atterberg et les essais de sédimentométrie sont réalisés selon les procédures des normes P 94-051 et P 94- 057, respectivement. Les essais de gonflement sous contrainte et de compres sion sont réalisés en compactant les échantillons à l'optimum Proctor normal. La teneur en eau optimale et la densité maximale de l'OPN ont été déterminées en utilisant la méthode de compactage normalisée par chocs décrite dans la norme P 94-093. Le compactage des échantillons humides est réalisẻ par compression dans des cellules cedométriques de $40 \mathrm{~mm}$ de diamètre sous une presse à la vitesse de $1 \mathrm{~mm} / \mathrm{min}$, jusqu'à obtenir la densité sèche fixée. A la fin du compactage les échantilons ont wne hauteur de $20 \mathrm{~mm}$. La pression de gonflement est mesurée dans les cellales cedométriques en utilisant deux méthodes, la première par humidification sous différentes contraintes imposées et la seconde par humidification à contrainte nulle suivie d'un chargement. Dans la première méthode, plusieurs échantillons compactés à l'OPN sont humidifiés par mise en contact avec un réservoir d'eau ( $u_{c}=0$ ), sous des contraintes mécaniques verticales variant de 10 à $1000 \mathrm{kPa}$ selon la procédure de la norme $\mathrm{P}$ 94-091. Dans la deuxième méthode, l"échantillon, initialement compacté aे l'OPN, est humidifié à pression capillaire nulle sous le poids du piston (correspondant à une contrainte de $10 \mathrm{kPa}$, désigné par la suite par « gonflement libre n) ; après stabilisation du gonflement, un chargement par paliers successifs de 10 à $1000 \mathrm{kPa}$ est appliqué à l'échantillon. Les essais sont suivis gräce à des capteurs de déplacement jusqu'” la stabilisation qui est atteinte quand le capteur de déplacement présente un taux de déplacement inférieur à 0,01 mm/jour.

Les essais de drainage-humidication à pression capillaire imposée $u_{\text {e }}$ et contrainte nulle ( $\sigma=0$ ) sont réalisés sur des échantillons préparés dans différents états iniliaux : (a) sous forme de pâte à une teneur en eau initiale de $1,5 \mathrm{w}_{1}$, (b) consolidés sous une contrainte de $100 \mathrm{kPa}$ dans un cedomètre et (c) compactés à l'OPN. Les pressions capillaires sont imposées en utilisant des plaques tensiométriques pour les pressions capillaires variant de $0,1 \mathrm{kPa}$ à $30 \mathrm{kPa}$, des solutions osmotiques pour les pressions capillaires varianl de 0,1 à 1,5 $\mathrm{MPa}$ et des solutions salines pour les pressions capillaires supérieures à 1,5 MPa (Biarez et al., 1988). Le principe des plaques tensiométriques est de mettre les échantillons en contact avec un réservoir d"eau maintenu en dépression par une colonne d'eau. Cet essaj nécessite d'utiliser des membranes de séparation semi-perméables qui permettent le passage de l'eau tout en empêchant le passage de l'air, ce qui est réalisé en pratique en utilisant un milieu poreux de falble porosité (verte fritté). En ce qui conceme l'osmose, les pressions capillaires sont imposées en utilisant des solutions de polyéthylène glycol (PEG de poids moléculaire 20000 ). Dans cette méthode, les échantillons sont mis au contact de 
la solution de PEG à travers une membrane de dialyse (diamètre des pores inférieur à $50 \mathrm{~nm}$ ) qui laisse passer l'eau et empêche le passage des molécules de PEG. L'eau est échangée entre la solution et le sol jusqu'à ce quu'il s'établisse un équilibre pour lequel la pression capillaire de l'eau dans l'échantilion correspond à Ja pression osmotique imposée par la concentration en PEG de la solution (Indarto. 1991). Les solutions salines, enfin, permettent d'imposer des pressions capillaires supérieures à $1.5 \mathrm{MPa}$. Le principe de cette méthode est d'utiliser des solutions salines qui, selon le sel utilisé et sa concentration, permettent d'imposer une valeur donnée d'humidité relative dans le récipient fermè où est placé l'échantillon. Il s'établit alors un équilibre entre la pression capillaire dans le sol et la pression de vapeur du liquide (loi de Kelvin).

Les diffractogrammes de rayons $X$ sont réalisés à l’air ambiant sur des échantillons lamellaires à faces parallèles coupés dans la direction paraltèle au sens de la contrainte appliquée. Les diffractogrammes sont enregistrés dans un intervalle d'angle quj varie de 2,8 à $70^{\circ}$, avec un pas de $0,02^{\circ}$ par seconde, ce qui correspond à une durée d'essai de $1 \mathrm{~h}$. Les diffractogrammes sont obtenus au moyen d'un appareil Siemens de type D5000 comportant un générateur de rayons $\mathrm{X}$ alimenté sous une tension de $35 \mathrm{kV}$ et une intensité de $30 \mathrm{~mA}$, muni d'un tube de rayons $X$ dont la cathode est en cuivTe $\left(\lambda_{\mathrm{Cuk}]}=0,15418 \mathrm{~nm}\right)$. En ce qui concerne les courbes d'analyse thermogravimétrique (ATG), les essais sont réalisés avec un appareil de type Setaram TGA 92 dont le gaz vecteur est l'argon et la vitesse de chauffage est de $5^{\circ} \mathrm{C} / \mathrm{min}$, dans une plage de température de $21^{\circ} \mathrm{C}$ aे $1000^{\circ} \mathrm{C}$. La masse des échantillons utilisés est d'environ $50 \mathrm{mg}$. Les mesures de porosimétrie au mercure sont effectuées sur des échantillons préalablement lyophilisés, selon la technique décrite par Tessier et Berrier (1979), de façon à préserver leur texture lors de la dessiccation. La porosimétrie au mercure est réalisée au moyen d'un porosimètre Micromeritics Autopore IV grâce auquel on peut appliquer des pressions variant de 0,0035 à $210 \mathrm{MPa}$, ce qui permet d’étudier des talles de pores variant de 350 um à 0,0036 pm. La taille des pores est déduite de la relation de Washburn (1921):

$$
P=\frac{2 \gamma \cos (\theta)}{r}
$$

où P est la pression d'injection du mercure, $\gamma$, ka tension superficielle du mercure (484 $\mathrm{mN} / \mathrm{m}), \theta$, l'angle de contact mercure/solide/air ( $130^{\circ}$ ) et $\mathrm{r}$, le rayon d'entrée des pores.

\section{Identification géotechnique et minéralogique des échantillons}

Le matériau utilisé provient de l’̂́le volcanique de Kimolos dans la mer Égée (Grèce). D'après Christidis et Scott (1997), les couches d'argile daterajent du Pléistocène (entre le Pliocène et l'Holocène). Cet échantillon, désigné ci-après par u argile grecque 0 , a été commercialisé par la société Sobrep. Du point de vue minéralogique, le diffractugramme de rayons X présente une première réflexion à $1,5 \mathrm{~m}$ caractéristique d'une montmorillonite calcique (Fig. 1). La bande (060) à $0,149 \mathrm{~nm}$ est révélatrice du caractère dioctaédrique de l'argile.

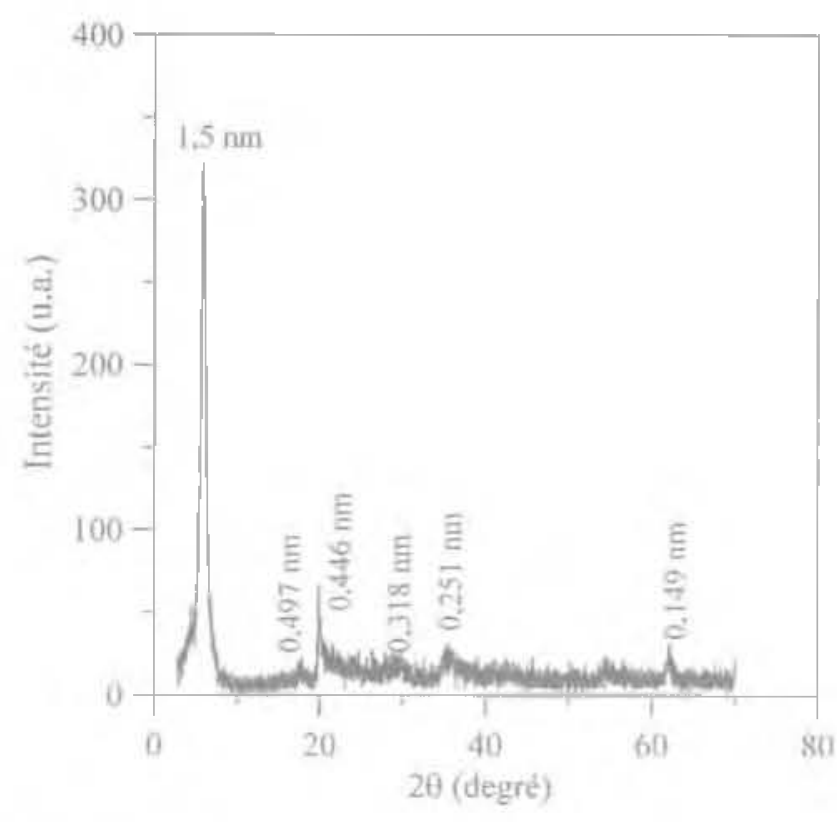

m5. 1 Diffractogramme de rayons $\mathbf{x}$ de l'argile grecque en poudre.

$X$-ray diffractogram of greek clay powder.

Les propriétés géotechniques de l'échantillon d'argile sont présentées dans le Tableau I (Fleureau et al. 1992). Les résuitats de sédimentométrie montrent que le matériau a un pourcentage de grains inférieurs ả $2 \mu \mathrm{m}$ de $40 \%$. Les limites de liquidité et de plasticité de cette argile sont respectivement égales ả 170 et $60 \%$. Ces données, reportées dans les abaques de Dakshanamurphy et Raman (1973), Chen (1988), Seed et al. (1962), Williams et Donaldson (1980), Building Research Establishment (1980) montrent que l'argile grecque est une argile très plastique à très fort potentiel de gonflement et de retrait (Fig. 2). A l'OPN, la masse volumique sèche, la teneur en eau et l'indice des vides sont respectivement de $1,15 \mathrm{Mg} / \mathrm{m}^{3}, 40 \%$ et 1,45 . Ces valeurs sont conformes à celles déduites des corrélations avec la limite de liquidité de l'argile (Fleureau et al., 2002).

TANLEA II Caractểistiques géotechniques de l'argile grecque.

Geotechnical properties of the greek clay.

\begin{tabular}{|c|c|c|c|c|c|c|c|}
\hline \multicolumn{3}{|c|}{ Granulometrle } & \multicolumn{3}{|c|}{ Plasticité } & \multicolumn{2}{|c|}{$\begin{array}{l}\text { Compactage } \\
\text { Proctor } \\
\text { normal (OPN) }\end{array}$} \\
\hline $\begin{array}{c}<80 \text {, } \\
(\%)\end{array}$ & $\begin{array}{c}<2 \mu m \\
(\%)\end{array}$ & $\begin{array}{l}d_{s 0} \\
(\mathrm{wm})\end{array}$ & $\begin{array}{l}w_{4} \\
(\%)\end{array}$ & $\begin{array}{l}w_{p} \\
{[\%)}\end{array}$ & $\begin{array}{c}\mathrm{t}_{p} \\
(\%)\end{array}$ & $\begin{array}{c}w \\
(\%)\end{array}$ & {$\left[\mathrm{kN} / \mathrm{m}^{3}\right)$} \\
\hline 100 & 40 & 4 & 170 & 60 & 110 & 40 & $1 t$ \\
\hline
\end{tabular}

\section{4 \\ Pression de gonflement des échantillons compactés à l'OPN}

Les mesures de la pression de gonflement sous charge constante et par gonflement libre suivi de rechargement sont présentées sur la figure 3 . Les valeurs obtenues sont de $540 \mathrm{kPa}$ pour la première méthode et de $880 \mathrm{kPa}$ pour la seconde. Cet écarl est en accord avec les résultats trouvés par Guiras-Skandaji (1996) qui a montré que l'essai de gonflement librerechargement conduisait à une valeur de pression de gonflement plus importante que l'essai à charge 

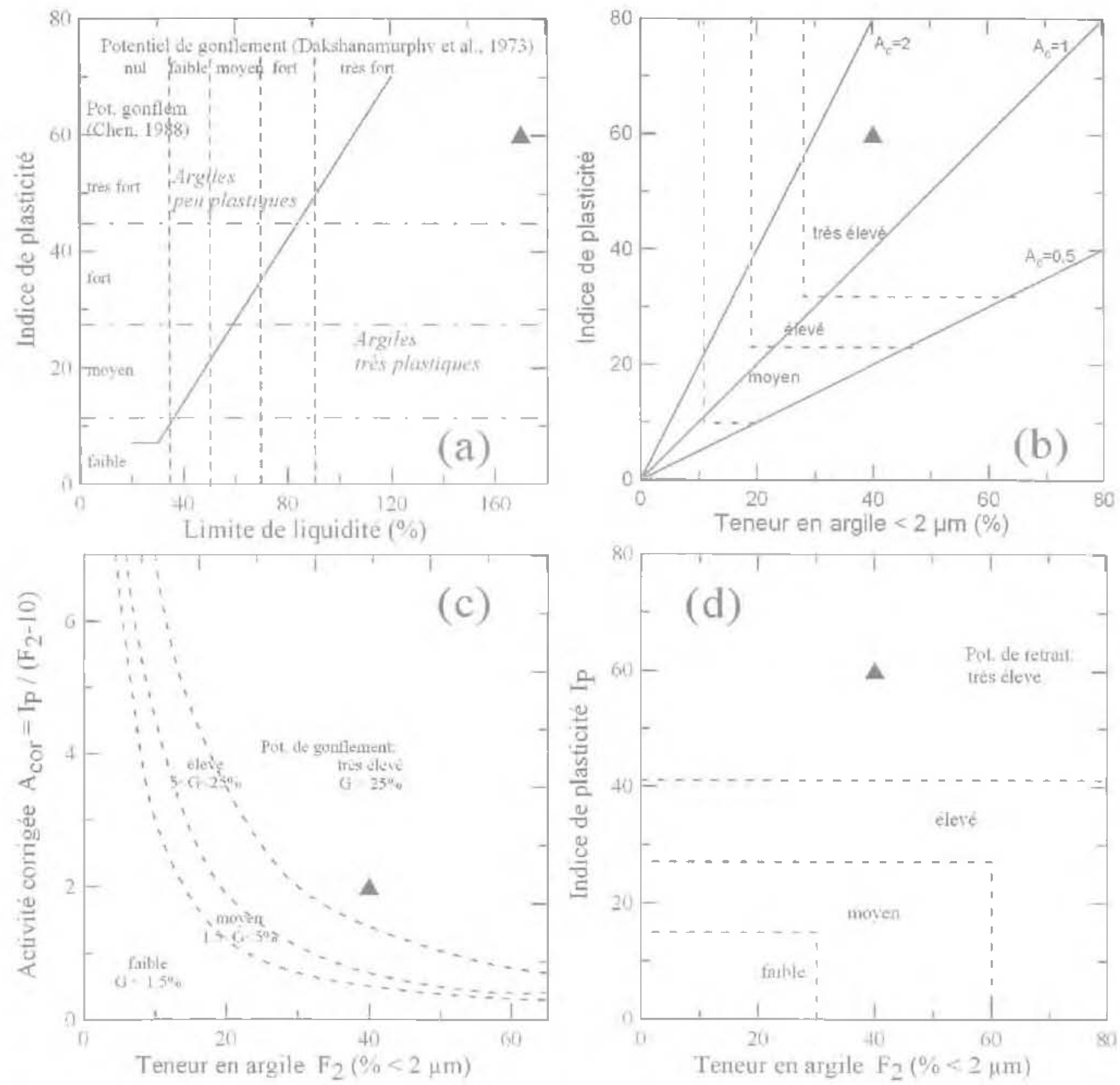

Fe. Classification du potentiel de gonflement ou de retrait de l'argile grecque en fonction des parametres d'identification (a) Dakshanamurphy et Raman, 1973 et Chen, 1988; (b) Williams et Donaldson, 1980 ; (c) Seed et al., 1962; (d) Builiding Research Establishment, 1980.

Classification of the swelling or shrinkage poteritial of the greek clay as a function of the identification parameters.

constante. Cette différence est évidemment liée aux déformations plastiques subies par le sol lors de la phase de gonflement libre gui entraĩnent une déstructџration plus ou moins forte de celui-ci. Souli (2006) a montré qu'au cours du gonflement libre, les particules tendent à s'orienter parallèlement les unes aux autres (cf. \& 6.1), phénomène qui s"accentue lors du chargement du matériau quasi-saturé. En revanche, lors de l'humidification d'un sol à volume constant, celui-ci tend à conserver la même texture que celle qu'il avait dans son état initial compacté, c'est-à-dire un arrangement plus isotrope des particules. Par conséquent, dans le premicr cas, les forces de répulsion de doublecouche seront plus élevées que dans le second cas, ce qui se traduira par une pression de gonflement plus forte. Pour la mesure sous charge constante, les conditions de déformation sont intermédiaires entre les deux cas précédents, surtout au voisinage de la pression de gonflement, ce qui explique la valeur mesurée de $540 \mathrm{kPa}$ par rapport aux $880 \mathrm{kPa}$ obtenus par gonflement-rechargement. Dans les essais de compression, il est utile de tracer la droite unormalement consolidée » (NC) qui représente le comportement d'ur matériau initialement peu dense : il s'agit donc d'une référence qui indique la compressibilité maximum que peut avoir le matériau saturé. La pente $\mathrm{C}_{\mathrm{c}}$ de la droite normalement consolidée est une caractẻristique intrinsèque du sol, reliée par exemple aux limites d'Atterberg. Biarez et Favre (1975) ont montré qu'on pouvait la définir, pour un sol saturé, à partir des points suivants :

$$
\begin{gathered}
w=w_{L} \text { soit } e=G_{s} w_{L} \text { pour } \sigma=7 \mathrm{kPa} ; \\
w=w_{p} \text { soit } e=G_{s} w_{p} \text { pour } \sigma=1000 \mathrm{kPa} ;
\end{gathered}
$$

où $\mathrm{G}_{\mathrm{s}}$ est la densité des grains solides, $w_{\mathrm{L}}$ et $\mathrm{w}_{\mathrm{p}}$ les limites de liquidité et de plasticité, respectivement. 
On constate sur la figure 3 que, dans le cas de l'argile grecque, la contrainte appliquée à la fỉn de l'essai de compression n'est pas suffisante pour que le matériau rejoigne le comportement normalement consolidé, il reste dans un état légèrement surconsolidé.

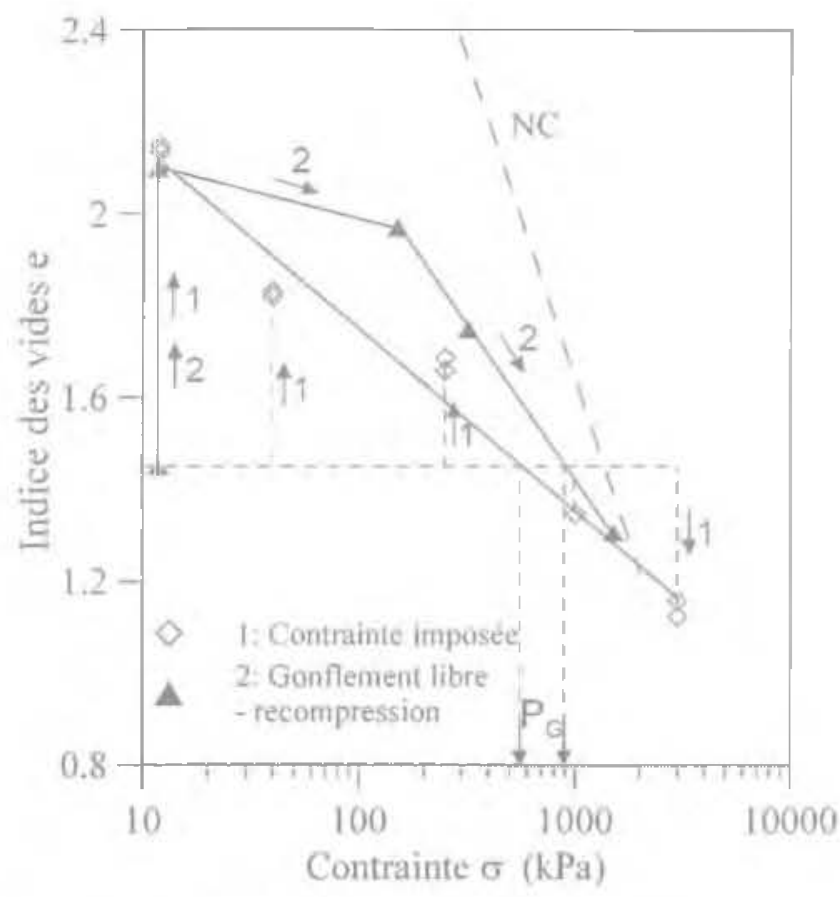

คล. 3 Mesure de la pression de gonflement de largile grecque compactée à l'optimum Proctor normal (1) par humidification sous charge constante et (2) par gonflement librerecompression : NC: Droite a normalement consolidée $n$.

Measurement of the swelling pressure of the greek clay compacted to Proctor optirnum derived from the (1) wetting under constant stress and (2) free swell-recompression methods; NC: 'normally corsolidated" line.

\section{Chemins de drainage-humidification}

\section{Échantillon normalement consolidé}

L'état nornalement consolidé est utilisé dans la présente étude comme référence pour comparer le comportement des échantillons compactés et surconsolidés. La figure 4 présente le premier cycle de drainage-humidjfication réalisé sur l'argile grecque sous forme de pâte à une teneur en eatu égale à une fois et demie la limite de liquidité Les chemins sont représentés dans les plans $\left[u_{r}, e\right]$, [ $w$, e] et $\left[u_{c}, S\right]$, où u représente la pression capillaire, e l'indice des vides, w la teneur en eau et $S_{r}$ le degré de saturation.

Dans le plan $\left[u_{c}\right.$, e], l'indice des vides initial de la pâte correspond au point $\mathrm{A}$, le chemin de drainaye (augmentation de $u_{r}$ j correspond à la branche de courbe AC. Cette partie de la courbe est elle-même đivisée en deux parties, la première notée $\mathrm{AB}$ et la seconde $\mathrm{BC}$. La partie $\mathrm{AB}$ met en évidence une diminution impor- tante de l'indice des vides alors que la courbe $\left[u_{v^{\prime}} S_{p}\right]_{\text {, }}$ montre que, pour cette gamme de pressions capillaires, l'échantillon reste saturé. A partir du point B, une faible variation de l'indice des vides est observée, qui correspond à une diminution importante du degré de saturation comme le montrent les courbes [u, $\left.\mathrm{S}_{\mathrm{r}}\right]$ et $\left[\mathrm{w}, \mathrm{S}_{\mathrm{r}}\right]$. En d'autres termes, lors du drainage, l'échantillon présente une première phase dans laquelie il se déforme et se réorganise de façon importante sans se désaturer, puis une seconde phase où il se désature fortement avec une falble déformation. La branche de courbe $\mathrm{AB}$ correspond à un comportement normalement consolidé du sol saturé ou quasi-saturé, ce qui signifie qu'elle peut être approchée par les corrélations en fonction de la limite de liquidité présentées dans la section 4 (Fleureau et al, 1993). Les lignes nomalement consolidées déduites des corrélations pour les différentes valeur's de w ont été tracées sur la figure 4 et l'on constate un bon accord entre la courbe de l'argile grecque et la ligne correspondant à sa limite de liquidité. La variation de l'indice des vides en fonction de la pression capillaîre est analogue à la courbe de compressibilité du sol, mais diffère de celle-ci par la présence d’un palier représenté par la branche BC. L'existence de ce palier permet de définir une valeur caractéristique de la pression capillaire qui marque la transition entre deux types de comportement très différents : un comportement saturé ou quasi-saturé « classique $»$ ( $\mathrm{Sr}>80 \%$ environ), dans lequel les concepts des sols saturés restent largement applicables, et un comportement non saturé sensiblement différent du précédent. Dans le cas de cette argile, la pression capillaire seuil est égale à $1000 \mathrm{kPa}$. Fleureau et al. (1992) ont montré que cette valeur dépendait de la minéralogie de l'échantillon et qu'elle étail importante dans le cas des smectites. Pratiquement, cette transition correspond à la limite de retrait, qui est estimée à $33 \%$ en utilisant la courbe [w, e].

La courbe d'humidification (branche CE) est ellemême divisée en deux parties, notées CD et DE. Les branches BC et CD sont pratiquement superposées. Comme la branche BC, la branche CD est caractérisée par une variation importante du degré de saturation, alors que la variation de l'indice des vides est très faible. Dans ce domaine de pression, on peut imaginer qu'il existe des ménisques d'eav entre les particules ou groupes de particules, qui se traduisent par des forces d'attraction très importantes et contribuent à l'augmentation de la rigidité et de la résistance du matériau mais sans provoquer de réorganisation puisque les forces intergranulaires sont peu inclinées par rapport aux plans de contact entre les grains (Biarez et al., 1993). On remarcue que sur les branches de courbes $A B$ et $D E$, les déformations sont fortement irréversibles. Sur cette partie de la courbe le sol est quasi saturé et l'échantillon présente un comportement surconsolidé.

\section{2}

\section{Échantillon compacté à l'OPN}

La Figure 5 présente be chemin de drainage-humidification du sol compacté à l'optimum Proctor normal. Dans ce cas, Péchantillon compacté possède une pression capillaire initiale qui augmente avec la limite de liquidité du sol et qui, pour l'argile grecque, est de l'ordre de 2 à $3 \mathrm{MPa}$ (représentée par la ligne en tirets épais sur la figure 5). Des corrélations ont également été établies entre ce paramètre et toutes les caractéristiques 

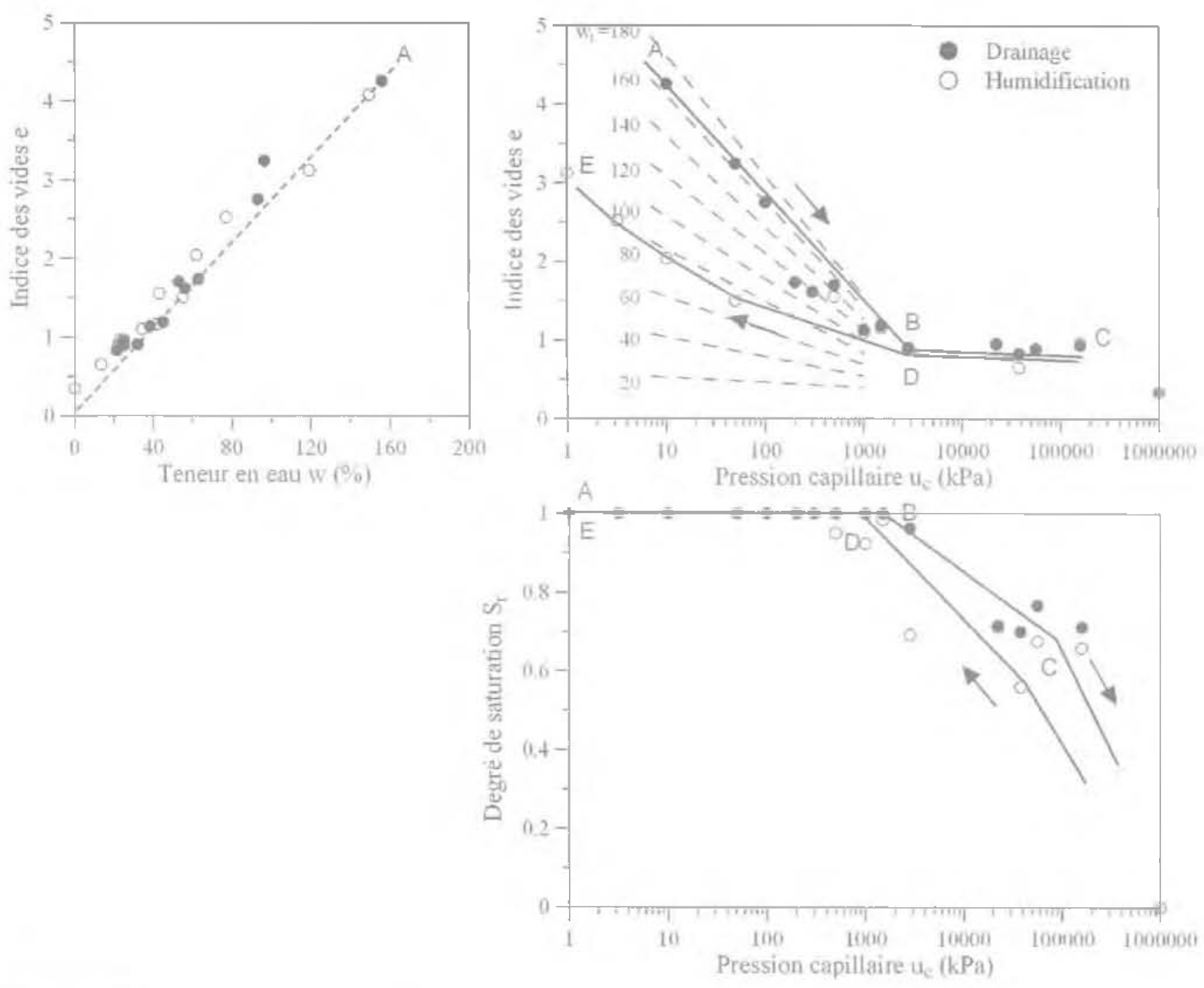

The 4 Courbe de drainage humidification de l'argile grecque sous forme de páte (wi = 1,5 wL) et comparaison avec le chemin normalement consolidé déduit des corrélations avec la limite de liquidité.

Drying-wetting curve of the greek clay prepared as a slurry (wi = 1,5 wL) and comparison with the normally consolidated path derived from the correlations with the liquid ఏirnit.

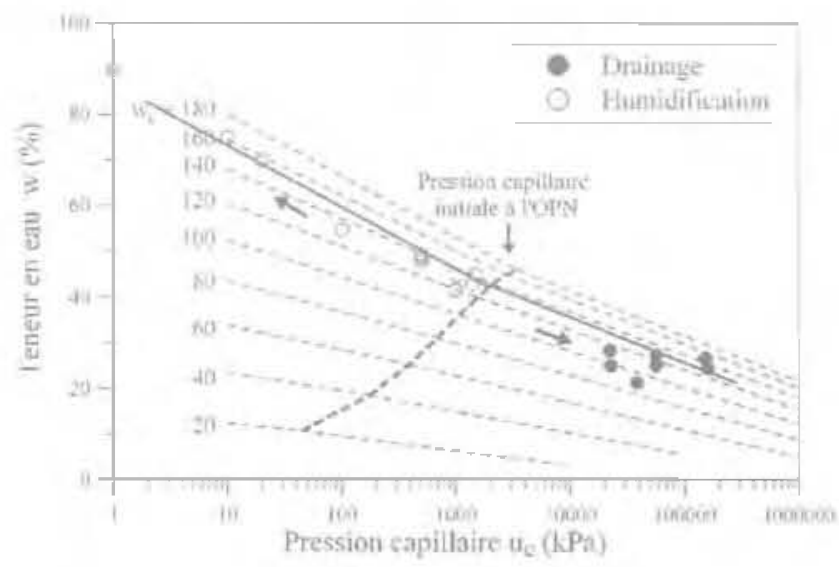

Fc.s Chemins de drainage et d'humidification de l'échantillon compacté à l'optimum Proctor normal et comparaison avec les corrélations déduites de la limite de liquididité.

Drying and wetting paths of the specimen compacted to the Proctor optimum and comparison with the correlations with the liquid limit. des chemins de drainage-humidification : indice des vides, teneur en eau et pression capillaire à l'OPN, pentes des chemins de drainage et d'humidification depuis l'OPN (Fleureav et al., 2002, 2003). Ces corrélations sont reportées sur la figure 5 pour les différentes valeurs de $W_{1}$. Là encore, on constate un bon accord entre les points expérimentaux de l'argile grecque et la ligne comespomdant à $w_{5}=170 \%$. D'une façon plus gếnérale, les corrélations permettent de donner une première estimation des chemins de drainage et d'humidification des sols compactés.

\section{3}

\section{Comparaison des chemins de drainage et d'humidification du sol compacté et des sols normalement consolidés et surconsolidés}

La figure 6 montre l'effet de l'état initial sur le chemin de drainage. Dans ce but, les échantillons ont été préparés dans trois état initiaux: (a) sous forme de pâte à une teneur en eau initiale de 1,5 fois la limite de liquidité, (b) consolidés sur chemin cedométrique 

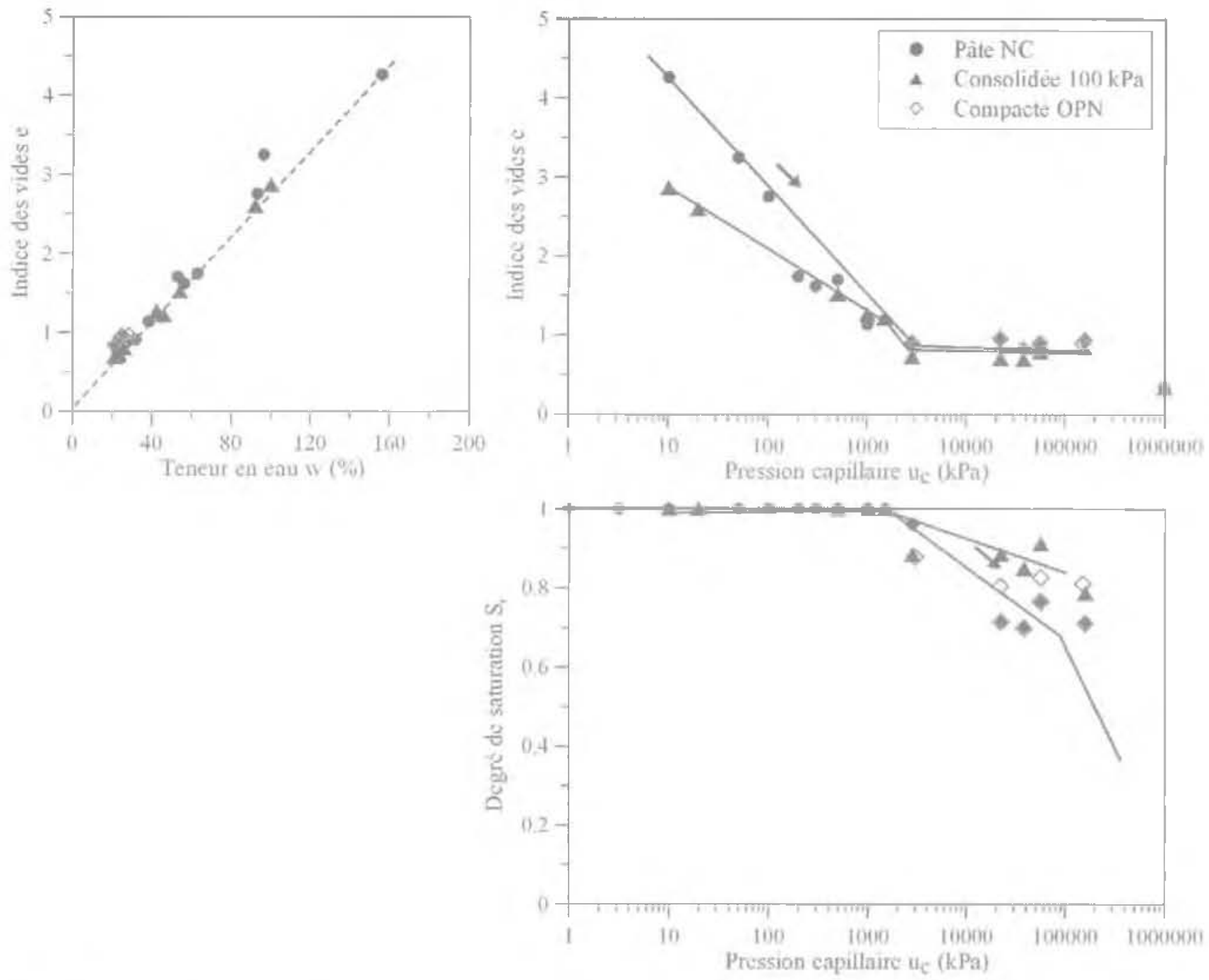

ค6.6 Effet de l'etat initial sur les courbes de drainage.

Effect of the initial state on the drying curves.

sous $100 \mathrm{kPa}$ de contrainte verticale et (c) compactés à l'OPN. La pâte consolidée à $100 \mathrm{kPa}$ présente le comportement d'un sol surconsolidé. Son chemin de drainage rejoint le chemin normalement consolidé dans le domaine saturé et présente le même palier et lá même limite de retrait. En ce qui concerne l'échantillon compacté à I'OPN, son chemin de drainage rejoint la courbe normalement consolidée dans le domaine non saturé mais on ne note pas de différence appréciable entre le palier de retrait du sol compácté et celuj de l'argile normalement consolidée. On peut donc dire en première approximation que le sol compacté à l'OPN se comporte de façon macroscopique comme un sol surconsolidé.

L'effet de l'état initial sur les chemins d'humidification des mêmes échantillons est présenté sur la figure 7 . Les résultats montrent que les trois matériaux présentent des chemins légèrement différents: le gonflement le plus important est observé pour la pâte séchée et la pâte consolidée séchée alors que la déformation apparaît sensiblement plus faible dans le cas đu sol compacté, humidifié à partir de son état initial, c'est-à-dire n'ayant pas subi de séchage. On retrouve là un résultat classique selon lequel le gonflement est d'autant plus important que la contrainte de consolidation du sol est plus forte. Dans le cas des deux échantillons séchés, cette contrainte de consolidation provient principalement de la pression capillaire de séchage, c'est-à-dire de l'écrouissage hydrique. On peut noter également que l'essentiel du gonflement des sols séchés se produit lorsque les matériaux se resaturent, c'est-à-dire pour des valeurs de pression capillaire inférieures à $2 \mathrm{MPa}$. L'échantillon compacté, en revanche, se resature beaucoup plus difficilement. Les différences observées entre l'échantillon compacté et les échantillons sẻchês, sur chemın d'hundifitication mais aussi, dans une certaine mesure, sur chemin de drainage, résultent probablement d'une texture différente liée au mode de préparation des matériaux. Il est en effet connu (Cui, 1993) que Ie mélange de particules humides et leur compactage conduit à la formation d'agrégats (ou a mottes $)$ ), tandis que la consolidation d'une pâte se traduit par des particules plus petites et une texture plus homogène et plus continue. Pour étudier plus en détail cet aspect, une étude associant différentes méthodes physico-chimiques a été réalisée en vue de caractériser les transformations texturales, de porosité et de répartition de l'eau du sol compacté lors de l'humidification. Des études similaires ont été effectuées par Qi (1996) sur des sols normalement consolidés. 

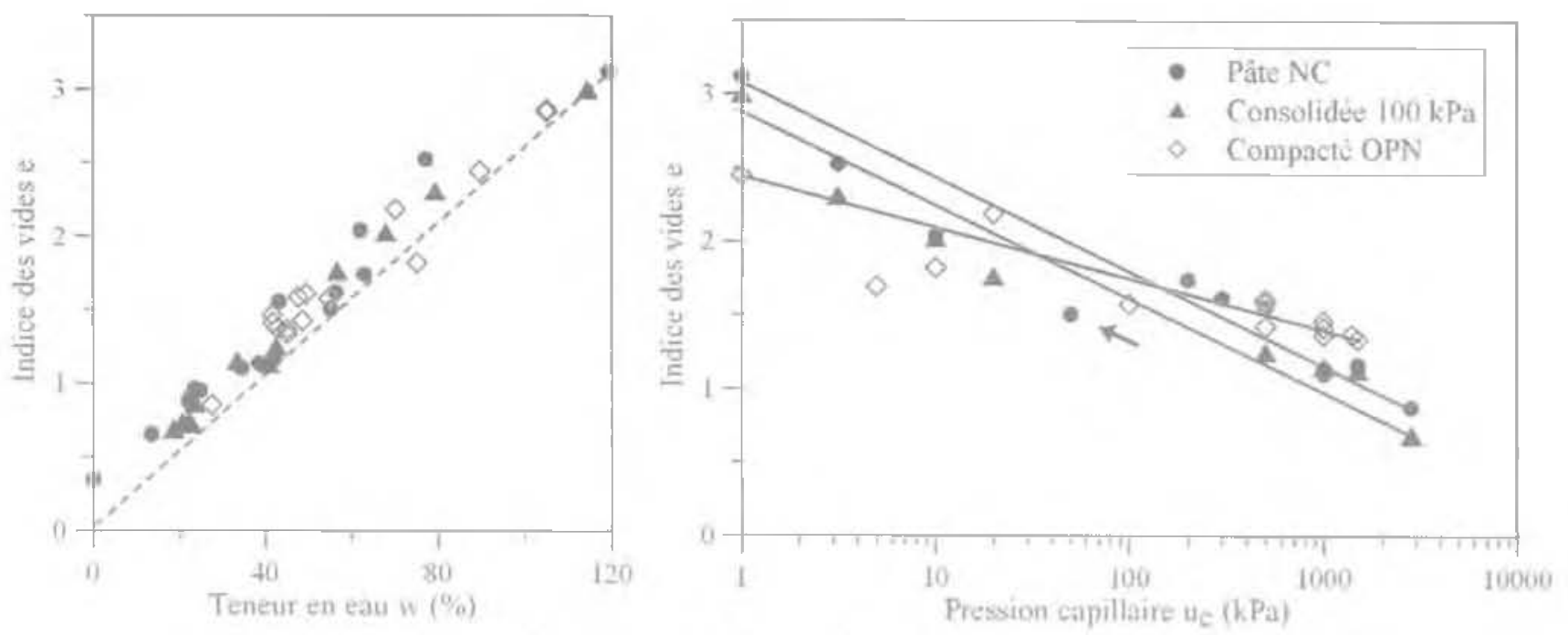

$\diamond$

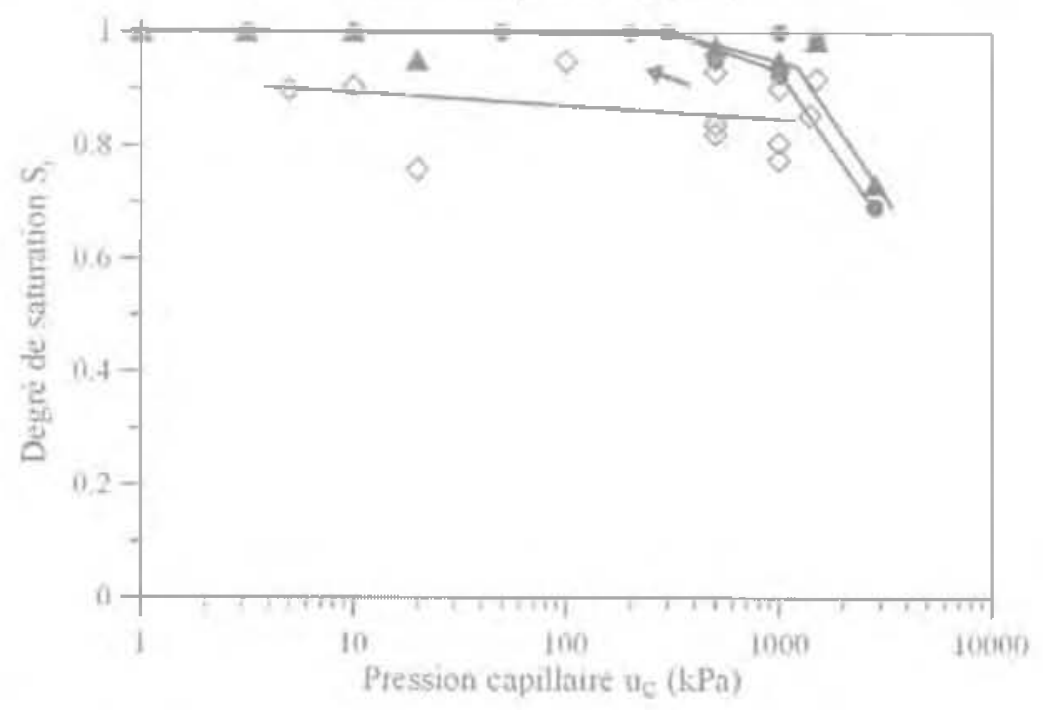

FG. 7 Effet de l'état initial sur les courbes d'humidification.

Effect of the initial state on the weting curves.

\section{6}

\section{Évolution des paramètres texturaux de l'échantillon compacté à l'OPN lors de l'humidification}

\section{1}

\section{Étude de l'hydratation par la diffraction des rayons $X(D R X)$}

La figure 8 présente les diffractogrammes de rayons $\mathrm{X}$ de l'argile grecque, à I'OPN et humidifiée en gonllement libre et sous une contrainte de $1000 \mathrm{kPa}$. L'étude des diffractogrammes permet de suivre l'évolution de l'état d'hydratation des échantillons par l'intermédiaire des modifications de la distance entre les feuillets d'argile (distance interfoliaire). L'échantillon compactê à l'OPN présente une distance interfoliaire de $1,58 \mathrm{~nm}$. Après humidification en gonflement libre, la distance interfoliaire se déplace à $1,90 \mathrm{~nm}$, ce qui correspond à l'insertion d'une couche d'eau par rapport à l'état initial. Sous la contrainte de $1000 \mathrm{kPa}$, en revanche, la distance interfoliaire varie peu puisqu'elle passe de 1,58 à 1,45 nm, mais il se produít une réorganisation des molécules d'eau dans l'espace interfojaire. Le nombre de couches d'eau est estimé en considérant que l'espacement entre les feuillets est de 0,9 à $1,0 \mathrm{~nm}$ et l'épaisseur d'une couche d'eau, de 0,25 à 0,30 nm. L'étude de l'intensité des réflexions (001), сотrespondant au premier pic de la courbe, permet d'avoir une idée sur l'évolution de l'organisation du matériau solus l'effet de la contrainte. En effet, l'échantillon est d'autant plus ordonné que l'intensité de sa réflexion (001) est importante (Guillot et al., 2001a). Dans le cas de l'argile grecque, l'échantillon humidifié en gonflement libre présente une intensité de réflexion (001) plus importante que celle de l'échantillon à l'OPN, ce qui traduit une augmentation de l'oricntation préférentielle des particules, c'est-â-dire de l'anisotropie du matériau, par rapport à celle de l'échantillon à l'état initial. Ce résultat peut s'expliquer par le fait qu'à l'état inttial, la pression capillaire relativement forte qui règne dans l'échantillon (2 à $3 \mathrm{MPa}$ ) crée des forces interparticulaires d'allraction qui śopposent à la réoryanisation du sol. Lors de l'humidification, la pression capillaire et les forces dues à l'eau s'annulent, ce qui permet un réarrangement des particules perpendiculairement à la direction d'application de la contrainte. L'étude 
des bandes $(02,11)$ permet de confirmer cette analyse. Si l'on compare avec l'échantilion humidifié sous la contrainte de $1000 \mathrm{kPa}$, les particules de l'échantiłlon humidifié en gonflement libre sont plus ordonnées.

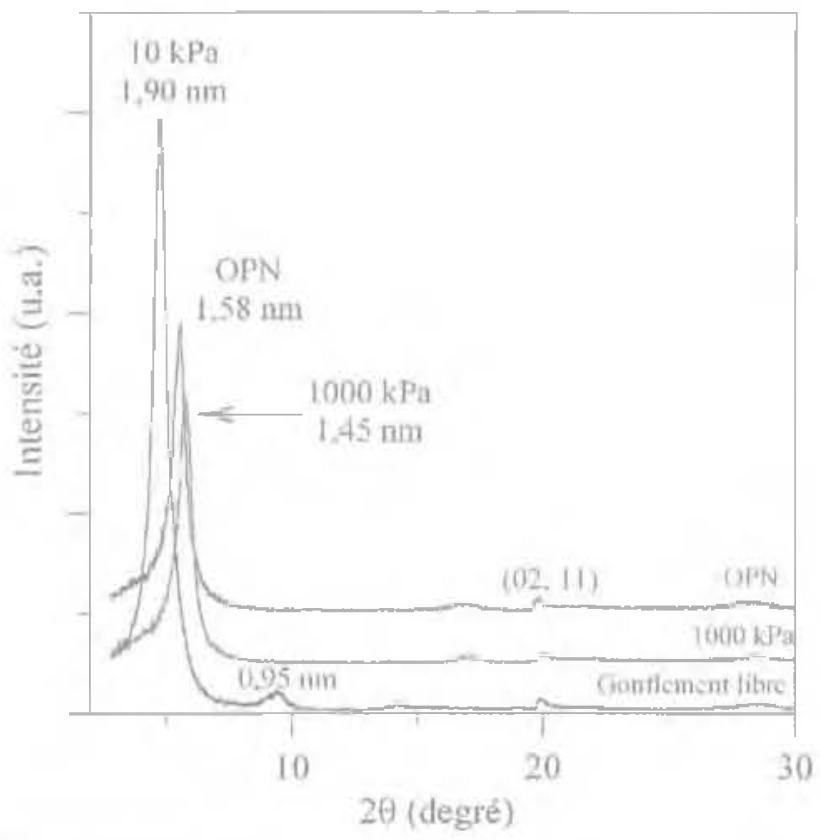

ne a Diffractogrammes de rayons $\mathrm{X}$ de l'argile grecque compactée à l'optimum Proctor normal et humidifié sans contrainte et sous une contrainte de $1000 \mathrm{kPa}$.

$\mathrm{X}$-ray diffractograms of the greek clay conpacted to Proctor optimum and hydrated without stress and under a saress of $1,000 \mathrm{kPa}$

\section{2}

\section{Étude de l'hydratation par analyse thermo- gravimétrique (ATG)}

La figure 9a présente les courbes d"analyse thermogravimétrique de l'argile grecque compactée à l'OPN.

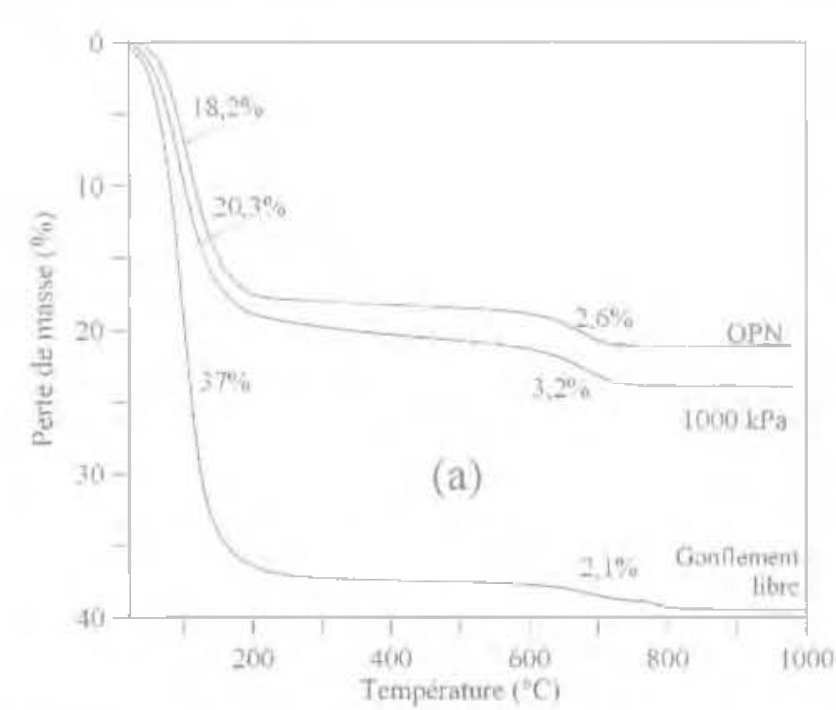

On remarque sur la courbe qu'il existe majoritairement deux types d'eau :

- l'eau hygroscopique qui est éliminée à une température comprise entre 110 et $600^{\circ} \mathrm{C}$. Cet intervalle de température correspond à l'élímination de l'eau faiblement liée entre 110 et $150^{\circ} \mathrm{C}$ et de l'eau plus fortement liée entre 150 et $600^{\circ} \mathrm{C}$. La quantité d'eau correspondant au dernier intervalle de température étant faible, elle a été intégrée dans le calcul à la quantité d'eau faiblement liée:

- l'eau structurale dont l'élimination correspond à la déshydroxylation des feuillets, à partir de $600^{\circ} \mathrm{C}$

Ia figure $9 \mathrm{~b}$ montre l'évolution des pourcentages d'eau fablement liée et de deshydroxylation en fonction de Ja contrainte mécanique. Les résultats indiquent que la quantitê d'eau faiblement liée, localisée dans jes espaces interagrégats, varie sensiblement avec la valeur de la contrainte mécanique. La comparaison des pertes d'eau, pour les températures inférieures a $200^{\circ} \mathrm{C}$, aux teneurs en eau des échantillons montre qu'une grande partie de la tenew en eatu totale est constituée d'eau faiblement liée. Ainsi, pour les échartillons compactés à l'OPN, l'eau faiblement liée constitue $46,9 \%$ de la teneur en eau totale de l'échantilion pour la contrainte de $1000 \mathrm{kPa}$ et $54,4 \%$ pour le gonflement libre. En ce qui concerne l'eau de déshydroxylation, elle varie peu et ne semble pas influencée par la contrainte mécanique.

\section{3}

\section{Porosité en fonction de la contrainte}

La variation des volumes cumulés en fonction de la taille des pores est présentée sur la figure 10. La courbe montre que l'augmentation de la contrainte sous laquelle s'effectue l'humidification se traduit globalement par la diminution du volume poreux. En réalité, le gonflement sous contrainte entraine une réorganisation de la distribution des pores; ainsi, les échantillons compactés à l'OPN présentent une répartiton continue des tailles de pores. Après le gonfiement libre, deux

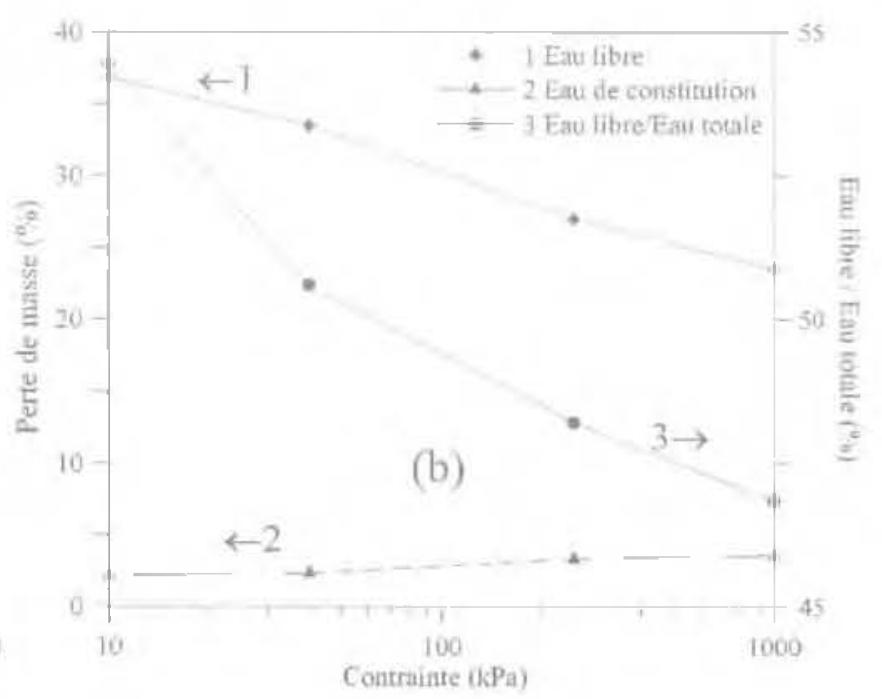

no.9 (a) Courbes thermogravimétriques du matériau compacté : (b) teneur en eau libre, teneur en eau de constitution et rapport de la teneur en eau libre à la teneur en eau totale à l'OPN et après hydratation en gonflement libre et sous une contrainte de $1000 \mathrm{kPa}$.

(a) Thermogravinetric curves of compacted clay; (b) free water content, constitution wafer content and ratio of fee water to total water contents at SPO and after hydration without stress and urder a stress of $1,000 \mathrm{kPa}$. 

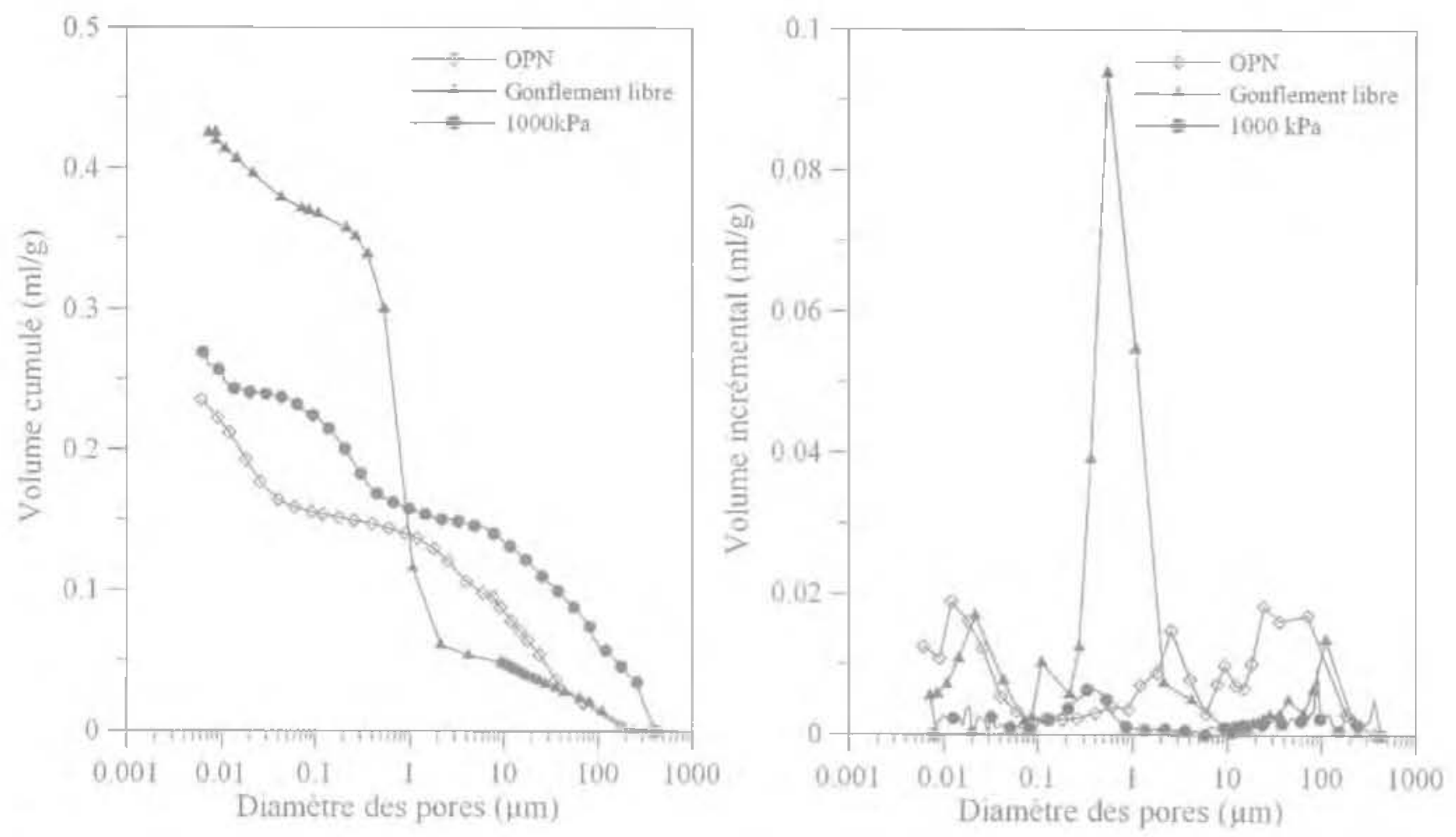

F0. 10 Volumes cumulés et incrémentaux en fonction du diamètre des pores à l'OPN et après hydratation en gonflement libre et sous une contrainte de $1000 \mathrm{kPa}$.

Cumulative and incremental volumes versus pore diameter at $\mathrm{PPO}$ and after bydration without stress and under a stress of $1,000 \mathrm{kPa}$.

familles de pores apparaissent, la première avec des diamètres proches de $100 \mu \mathrm{m}$ et la seconde, autour de $1 \mu \mathrm{m}$. Pour l'échantillon humidifié sous la contrainte de $1000 \mathrm{kPa}$, il apparaît une seute famille de pores, centrée sur 0,3 um. Le tableau II présente la contribution de la porosité déduite des essais de porosimétrie au mercure à la porosité totale calculée à partir de l'indice des vides. Pour les différents essais réalisés, il apparait que la porosimétrie au mercure permet d'accéder à 55 à $60 \%$ du volume des pores.

tasigau II Tableau II. Pourcentage de la porosité totale mesurée par porosité au mercure à l'OPN et après hydratation en gonflement libre ou sous une contrainte de $1000 \mathrm{kPa}$.

Percentage of total porosily measured by mercury intrusion porosimetry at SPO and after hydration without stress or under a stress of $1,000 \mathrm{kPa}$.

\begin{tabular}{|c|c|c|c|c|}
\hline & Contrainte & $\begin{array}{c}\text { Volume } \\
\text { total des } \\
\text { vides } \\
\text { (mi/g) }\end{array}$ & $\begin{array}{l}\text { Volume } \\
\text { de mercure } \\
\text { introduit } \\
\text { (ms/g) }\end{array}$ & $\begin{array}{c}\text { Pourcentage } \\
\text { de la porosité } \\
\text { mesurée par } \\
\text { porosimétrie } \\
\text { au mercure } \\
\text { (\%) }\end{array}$ \\
\hline \multirow{3}{*}{$\begin{array}{l}\text { Argile } \\
\text { grecgue } \\
\text { OpN }\end{array}$} & Etat initial & 0,54 & 0,22 & 41 \\
\hline & $\begin{array}{l}\text { Gonfte- } \\
\text { ment libre }\end{array}$ & 0,93 & 0,45 & 55 \\
\hline & $1000 \mathrm{kPa}$ & 0,44 & 0,26 & 60 \\
\hline
\end{tabular}

\section{4}

\section{Synthèse des résultats des mesures physico-chimiques}

Les résultats obtenus par la diffraction des rayons $X$, la porosimétrie au mercure et l'analyse thermique mettent en évidence la complexité d'interprétation du gonflement à l'échelle microscopique. En etfet, il ressort nettement de la porosimétrie au mercure et de l'analyse thermique que, dans l'argile compactée, ce sont essentieliement les macropores interagrégats qui contribuent aџ processus de gonflement. Par ailleurs, les résulats de la diffraction des rayons X suggèrent qu'il existe des pores dont le diametre est de l'ordre du nanomètre, quil jouent un rôle important dans le phénomène mais ne sont pas pris en compte par la porosimétrie au mercure. Ces pores sont influencés par la variation de la contrainte et de l'état d'hydratation des échantillons. Ainsi, l'eau s'organise dans l'espace interfoliaire en fonction de l'état d'hydratation des échantillons : pour les faibles contraintes, la quanlité de pseudo-couches d'eau dans l'espace interfoliaire augmente par rapport à l'état intial, alors que, pour l'échantillon humidifié sous des contraintes plus fortes ( $1000 \mathrm{kPa}$ dans le cadre de cette étude), les molécules d'eau se réorganisent dans l'espace interfoliaire puisqu'à l'état initial et sous la contrainte de $1000 \mathrm{kPa}$, les distances interfoliaires, respectivement égales à 1,58 et $1,45 \mathrm{~nm}$, correspondent au même nombre de couches d'eau. 
I] faut nuancer ces conclusions par le fait que les techniques utilisées dans cette étude ne permettent pas d'avoir accès à tous les pores du milieu, comme on le constate en calculant la somme des porosités déduites de la DRX el de la porosimétrie au mercure, quí reste inférieure à la porosité totale déduite de I'indice des vides global. En effet, il existe dans les argiles des mésopores, dont la taille est inférieure à $2 \mathrm{~nm}$, qui ne sont accessibles que par d'autres techniques physico-chimiques comme la BET (Brunauer et al., 1938). D’ailleurs, les courbes de porosimétrie des échantillons ne présentent pas de palier au niveau des petits pores (au dessous de 6,3 nm), ce qui semble confirmer l'existence de mésopores dont la tailla est inférieure à $6 \mathrm{~nm}$. Certains auteurs (Guillot et al., 2001b) ont également émis des critiques sur la technique de lyophilisation. utilisée pour la préparation des échantillons pour les mesures de porosimétrie au mercure, qui ne permettrait pas d'extraire toute l'eau, ce qui peut paraître logique compte tenu de la complexité de la texture des smectites. Il faut aussi tenir compte des pores occlus, qui ne sont pas accessibles mais qui pourraient constituer une part non négligeable de la porosité.

\section{7}

\section{Conclusion}

L'article présente quelques aspects du comportement d'une argile très plastique compactée sur les chemins de drainage et d'humidtication, en considérant à la fois les déformations macroscopiques et les changements à l'échelle microscopique. Dans une première partie, les variations d'indice des vides et de degré de saturation de l'argile compactée sont comparées à celles d'échantillons sous forme de pâte ou de pâte consolidée. Sur le chemin de drainage, l'échantỉlon compacté à l'optimum Proctor nomal présente un comportement analogue à celui d’un échantillon surconsolidé, avec un palier de retrait identique à celui de la pâte ou de l'échantillon consolidé sous une contrainte de $100 \mathrm{kPa}$. Lors de l'humidification, le chemin de l'échantillon compacté se distingue nettement de ceux de la pâte ou de la pâte consolidée séchẻes et l'échantillon présente des déformations plus faibles.
Cette différence peut probablement être attribuée à la texture " en agrégats 》 de l'échantilon compacté, très différente de la texture plus continue des autres échantillons.

Dans la seconde partie, une étude microscopique utilisant différentes techniques physico-chimiques a été réalisée sur l'évolution de la texture du matériau compacté pendant l'humidification afin d'expliquer le comportement observé macroscopiquement. La diffraction des rayons $X$ indique çue $1^{t}$ humidification entraîne une variation de la distance interfoliaire en gonflement libre et la réorganisation des molécules d'eau adsorbées dans l'espace interfoliaire sous une contrainte de $1000 \mathrm{kPa}$. Du point de vue de la texture, la comparaison des intensités des rélexions (001) montre que, lors de l'humidification, les particules s'orientent de façon préférentielle, d'autant plus que la contrainte est faible. On note ainsi une forte augmentation de l'anisotropie de l'échantillon lorsque la pression capillaire diminue. Les mesures d'analyse thermique et de porosimétrie au mercure révêlent que, dans le sol compacté, l'essentiel de l'eau se trouve dans les espaces interagrégats. Ainsi, les résultats de l'analyse themique suggèrent que la quantité d'eau libre varie linéairement en fonction de là contrafnte appliquée et que la quantité d"eau libre constitue environ $50 \%$ de la porosité totale des échantillons. Par ailleurs la porosité interagrégats peut occuper jusqu'à $60 \%$ de la porosité totale des échantillons. Le rôle important joué par les pores inter-agrégats au sein de l'argile compactée permet de comprendre les différences observées entre les échantillons compactés et consolidés. Toutefoiss, les conclusions doivent être nuancées par le fait que les techniques utilisées ne permettent pas de mesurer toute la porosité des échantillons comme les pores occlus ou les mésopores.

En conclusion, les méthodes physico-chimiques apportent des éléments complémentaires essentiels pour la compréhension de phénomènes complexes comme le retrait-gonflement des sols argileux. Elles permettent notamment d'avoir accès à l'évolution des différentes classes de porosité des échantillons, de la répartition de l'eau à l'intérieur de ces pores et de la texture (anisotropie) du matériau. 
Biarez J., Favre J.-L. - Parameters filing and statistical analysis of data in soil mechanics. $2^{\text {nd }}$ International Conference on Application of Statistics and Probabilities in Soil Mechanics. Aachen, vol. 2, 1975 , p. 249-264.

Biarez J., Fleureau J.-M., Zerhouni M.I., Soepandji B.S. - Variations de volume des sols argileux lors de cycles de drainage-humidification, Revue française de géotechnique. 41, 1988, p. 63-71.

Biarez J., Fleureau J.-M., Taibi S. - Mechanical constitutive model for unsaturated granular media made up by spheres. $2^{\text {nd }}$ International Conference on Micromechanics of Granular Media. Birmingham, Thornton (ed.), Rotterdam: Balkema, 1993, p. 51-58.

Biarez J., Fleureau J.-M., Taïbi S. -Critère de résistance maximale des sols non saturés : Approche expérimentale et modélisation, 13th International Conference on Soil Mechanics and Foundation Engineering, New-Delhi, January, Oxford \& I.B.H. Pub., 194, p. 385-388.

Brunauer S., Emmett P.H., Teller E. 1938. Adsorption of gases in multimolecular layers. Journal of the American Chemical Society, pp. 309-319.

Building Research Establishment. - The effect of a roof on a fire within a building. Rapport IP 3/80, 1980.

Chen F.H. 1988. Foundations on expansive soils. Developments in Geotechnical Engineering. Elsevier Publishing Co, Amsterdam, 54, 1988, $464 \mathrm{p}$.

Christidis G.E., Scott P.W. - The origin and control of colour of white bentonites from the Aegean islands of Milos and Kimolos, Greece. Mineralium Deposita. 32,1997 , p. 271-279.

Cui Y. J. - The unsaturated behaviour of a compacted silty clay. 7th Young Geotechnical Engineers Conference, Böbligen, 1993, p. 1.31-1.33.

Dakshanamurphy V., Raman V. 1973. A simple method of identifying an expansive soil. Soils and Foundations. 13, pp. 97-104.

Fleureau J.-M., Soemitro R., Taibi S. Behavior of an expansive clay related to suction. $7^{\text {th }}$ International Conference on Expansive Soils. Dallas, Aug. 1st 1992. p. $173-178$.

Fleureau J.-M., Kheirbek-Saoud S., Soemitro R., Taibi S. - Behaviour of clayey soils on drying-wetting paths. Canadian Geotechnical Journal. 30, $\mathrm{n}^{\circ} 2,1993$, p. 287-296.

Fleureau J.-M., Kheirbek-Saoud S., Taïbi S. - Experimental behaviour of unsatu- rated soils or soils with a negative pressure. 1st International Conference on Unsaturated Soils, Paris, Balkema ed., vol. 1, 1995, p. 57-62.

Fleureau J.-M., Verbrugge J.-C., Huergo P.J., Gomes Correia A., Kheirbek-Saoud S. -Description and Modelling of the Drying and Wetting Paths of Compacted Soils. Canadian Geotechnical Journal. 39, 2002, p. 1341-1357.

Fleureau J.-M., Hadiwardoyo S., KheirbekSaoud S. - Simplified approach to the Behaviour of Compacted Soils on Drying and Wetting Paths. $3^{\text {rd }}$ Int. Conf. on Unsaturated Soils. Recife (Brésil), 10-13 Mars 2002, J.F.T. Juca, T.M.P. de Campos \& F.A.M. Marinho (eds.), Balkema, Lisse, vol. 3, 2003.

Guillot X., Bergaya F., Fleureau J.-M., AlMukhtar M. 2001a. Influence of stresses and suction on volume change behavior and microscopic properties of a Ca-smectite. International Symposium on suction, swelling, permeability and structure of clays. Shizuoka (Japan), 2001a, p. 69-77.

Guillot X., Al-Mukhtar M., Bergaya F., Fleureau J.-M. - Estimation de la porosité dans un matériau argileux. Comptes-rendus de l'Académie des sciences, Géosciences, 334, 2001b, p. 105-109.

Guiras-Skandaji H. - Déformabilité des sols argileux non saturés : étude expérimentale et application à la modélisation. Thèse de doctorat, Institut national polytechnique de Lorraine, Nancy, 1996.

Hachichi A., Fleureau J.-M. - Caractérisation et stabilisation de quelques sols gonflants d'Algérie. Revue française de géotechnique, 86, 1999, p. 47-51.

Indarto. 1991. Comportement mécanique et hydrique des matériaux compactés et de remblais. Thèse de doctorat, École centrale Paris.

Komine H., Ogata N. - Experimental study on swelling characteristics of compacted Bentonite. Canadian Geotechnical Journal, 31, 1994, p. 478-490.

Modaressi A., Abou Bekr N, Fry J.-J. - Unified approach to model partially saturated and saturated soils. 1st international conference on unsaturated Soils, Paris, E.E., Alonso and Delage (eds), Rotterdam, Balkema, Paris, Presses des Ponts et Chaussées, vol. 3, 1996, p. 111116.

Norme française NF P 94-057 - Sols : Reconnaissance et Essais. Analyse granulométriques des sols. Méthode par sédimentation. AFNOR, 1992, $17 \mathrm{p}$.
Norme française NF P 94-051 - Sols: Reconnaissance et Essais. Détermination des limites d'Atterberg. Limite de liquidité à la coupelle - limite de plasticité au rouleau. AFNOR, 1993, $15 \mathrm{p}$.

Norme française XP P 94-091. - Sols : reconnaissance et essais. Essai de gonflement à I'œdomètre. Détermination des déformations par chargement de plusieurs éprouvettes. AFNOR, 1995, $13 \mathrm{p}$.

Norme française NF P 94-093. - Sols : reconnaissance et essais. Détermination des références de compactage $d^{\prime} u n$ matériau. Essai Proctor normal. Essai Proctor modifié. AFNOR, 1999, 18 p.

Qi Y. - Comportement hydromécanique des argiles : couplage des propriétés micromacroscopiques de la laponite et de l'hectorite. Thèse de doctorat, université d'Orléans, 1996.

Seed B., Woodward R.J., Lundgren R. Prediction of swelling potential for compacted clays. Journal of the Soil Mechanics and Foundation Division, American Society of Civil Engineers, vol. 88, SM3, 1962, p. 53-87.

Souli H. - Études hydromécanique et physico-chimique de deux argiles en présence de cations métalliques. Thèse de doctorat, École centrale Paris.

Suratman - Contribution à l'étude de la cinétique et de la stabilisation du gonflement des argiles. Thèse de docteuringénieur. INSA de Lyon, 1995.

Tessier D., Berrier J. - Utilisation de la microscopie électronique à balayage dans l'étude des sols; observations de sols humides soumis à différents $p F$. Science du sol, Bulletin de l'AFES 1, 1979, p. 67-82.

Washburn E.W. - Note on a method of determining the distribution of pore sizes in a porous material. Natural Academic Science Proceedings, vol. 7, 1921, p. 115-116.

Williams A.B., Donaldson G.W. - Developments related to building on expansive soils in South Africa. $4^{\text {th }}$ International Conference on Expansive Soils. Denver, vol. 2, 1980, p. 834-844 